\title{
Excitation Test Results on a Single Inner Vertical Coil for the Large Helical Device
}

\author{
K. Takahata, T. Mito, T. Satow, A. Nishimura, S. Yamada, H. Chikaraishi, N. Yanagi, A. Iwamoto, R. Maekawa, S. Imagawa, \\ H. Tamura, S. Yamaguchi, S. Satoh, S. Tanahashi, K. Yamazaki, J. Yamamoto, O. Motojima, and EXSIV Group \\ National Institute for Fusion Science (NIFS), Oroshi, Toki, Gifu 509-52, Japan \\ T. Kai, K. Nakamoto, T. Yoshida, Y. Wachi and M. Ono \\ Toshiba Corporation, Chiyoda, Tokyo 100, Japan
}

\begin{abstract}
Excitation experiments on a single inner vertical coil for the Large Helical Device (LHD) were carried out to confirm its performance. The coil is one of the LHD's poloidal field coils and consists of a forced-flow $\mathrm{Nb}$-Ti cable-in-conduit conductor (CICC). After coold own for 250 hours, the supercon ducting transition of the wh ole coil was con firmed. Pressure drops were measured during the cooldown to determine the coil's hydraulic characteristics. Then, the coil was successfully energized up to the specified current, $20.8 \mathrm{kA}$. In the exp eriments, heat gen eration of joints, radial displacement and acoustic emission (AE) were measured.
\end{abstract}

\section{INTRODUCTION}

The LHD is a heliotron type experimental fusion device which has been under construction since 1990 and will be completed in 1998 [1]. One of the special features of the LHD is that all coils, a pair of helical coils and three pair of poloidal coils, are superconducting. The poloidal coils consist of Inner Vertical (IV), Inner Shaping (IS) and Outer Vertical (OV) field coils.

We performed cooldown and excitation tests of an IV coil using test facilities at the cryogenics and superconductivity laboratories at the National Institute for Fusion Science (NIFS). The main purpose of this experiment was to confirm the coil's performance before installing it in the LHD cryostat. The other purpose was to demonstrate operations of a large forced-flow coil, including a cooling system, a helium circulating unit, a power supply, a superconducting bus line and a protection system. The first experiment was performed from February 1 to March 1, 1995. The coil was cooled down to the superconducting state for 23 days [2]. A dust filter in an inlet pipe was, however, blocked by impurities in helium gas. For this reason, an excitation test could not be carried out. A new filter system was developed and the capacity of the cooling system was increased. The second experiment was performed from November 13 to December 8 , 1995. The peripheral devices, such as the centrifugal pump and the superconducting bus line, could be operated smoothly, and the coil was successfully energized up to the LHD nominal current, $20.8 \mathrm{kA}$. In this paper, the obtained data during the cooldown and excitations are presented.

\section{CONDUCTOR AND COIL}

The conductor is a Nb-Ti CIC type. The specifications are listed in Table I. The number of strands is 486 and the void

Manuscript received August 27, 1996. fraction is 0.38 . The conductor is designed to have high stability. The surface of strands is therefore bare. The conductor is wrapped with $0.5 \mathrm{~mm}$ thick impregnated glass epoxy tape (glass fabric / polyimide / glass fabric laminates) and wound into eight double-pancake coils. The thickness of the insulator between pancakes is $1.5 \mathrm{~mm}$.

The specifications of the coil are listed in Table II. The operating current and the maximum field are $20.8 \mathrm{kA}$ and 5.2 T, respectively. Figure 1 shows a cooling flow diagram for the coil. The coil consists of 16 pancake coils, and coolant flows in parallel from inner turns to outer turns. The spiral pitch changes direction from one double pancake to the next to reduce error field due to layer to layer transition. Seven conductor joints are arranged outside and become the outlets of the coolant. In the joints, the solid state bonding technique, where the $\mathrm{Nb}-\mathrm{Ti}$ filaments are directly jointed, is applied to make the joints compact and to reduce error field $[3,4]$. The coolant returns through insulation pipes and a manifold. The length of a flow path is $170 \mathrm{~m}$ on average. The coil was wrapped with a ground insulator of $4 \mathrm{~mm}$ thickness and covered with ten fan-shaped stainless steel planks of $40 \mathrm{~mm}$ thickness. The planks are called "PC sleeve" and their roles are not only to fix the coil into the supporting shell of the LHD but also to shield the coil from heat loss by the installed cooling pipes.

\section{TEST PROCEDURE}

Instrumentation is also indicated in Fig. 1. The inlet and outlet gas temperatures (T) were measured using $\mathrm{Pt}-\mathrm{Co}$ and carbon-glass-resistor (CGR) thermometers in combination. Voltage taps (V) were attached to the pipes to measure the resistance of each pancake and joint. During the cooldown, the resistance was transformed into the average coil temperature. Flow orifices $(F)$ were installed into the inlet and outlet pipes. Pressure taps $(\mathrm{P})$ were also installed to measure the pressure drop.

The coil was set on ten cryogenic supporting posts in a single-coil-testing cryostat and can slide on Teflon surfaces on the posts. The coil was then connected to the test facilities including the helium refrigerator, the supercritical helium (SHe) centrifugal pump unit, the superconducting bus line and the $75 \mathrm{kA}-21 \mathrm{~V}$ power supply. The capacity of the helium refrigerator is $600 \mathrm{~W}$ at $4.4 \mathrm{~K}$ or $250 \mathrm{l} / \mathrm{h}$. The inlet gas temperature is controlled by mixing cold and hot gases from the cold box to keep the temperature difference between inlet and outlet less than $50 \mathrm{~K}$. When the coil temperature became $90 \mathrm{~K}$, turboexpanders in the cold box were started, and the coil was cooled by gas at $10-20 \mathrm{~K}$. After the initial 
TABLE I

SPECIFICATIONS OF THE IV CONDUCTOR

\begin{tabular}{ll}
\hline Conductor type & Cable-in-conduit \\
Superconductor & Nb-Ti \\
Conduit dimension & $23.0 \mathrm{~mm} \times 27.6 \mathrm{~mm}$ \\
\multicolumn{1}{c}{ thickness } & $3.0 \mathrm{~mm}$ \\
Void fraction & 0.38 \\
Strand diameter & $0.76 \mathrm{~mm}$ \\
Number of strands & 486 \\
NbTi:Cu ratio & $1: 2.7$ \\
Strand surface & Bare copper \\
\hline \multicolumn{1}{c}{ SPECIFICATIONS OF THE IV COIL } \\
& Supercritical He forced-flow \\
Cooling type & $3.6 \mathrm{~m}$ \\
Average diameter & $0.47 \mathrm{~m}$ \\
Height & $16 \mathrm{ton}$ \\
Total weight & $15 \times 16=240$ \\
Number of turns & $20.8 \mathrm{kA}$ \\
Operating current & $5.2 \mathrm{~T}$ \\
Maximum field & 16 \\
Number of cooling paths & $170 \mathrm{~m}$ \\
Cooling path length &
\end{tabular}

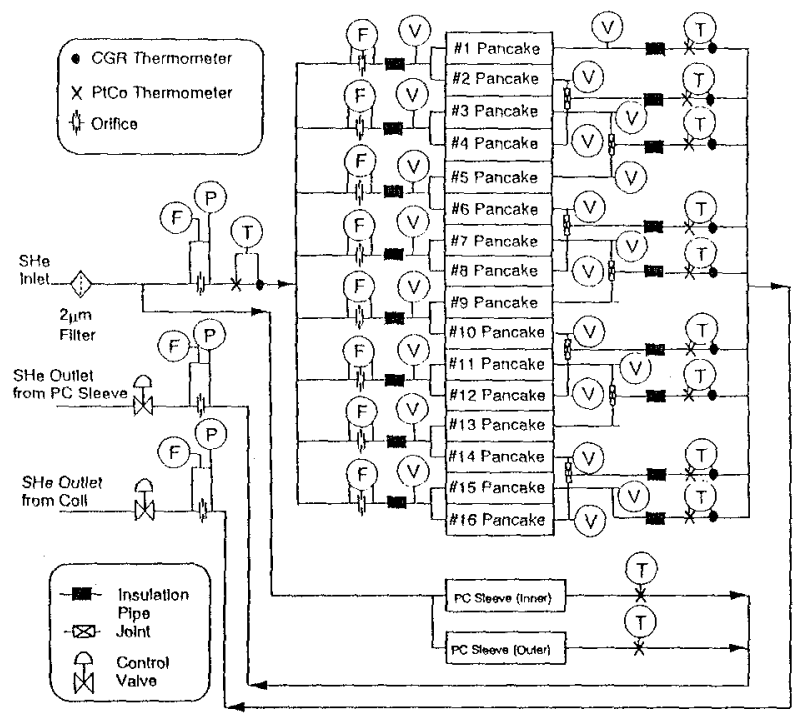

Fig. 1. Cooling flow diagram and instrumentation in the coil.

cooling, steady cooling was carried out by the SHe circulating system which can generate a mass flow up to $50 \mathrm{~g} / \mathrm{s}$ at $4.4 \mathrm{~K}$. Figure 2 shows the cooling flow diagram of the circulating system. The SHe circulates though the pump, the heat exchanger with liquid helium (LHe) and the coil. A stainless steel filter with a mesh size of $5 \mu \mathrm{m}$ is installed between the pump unit and the coil cryostat to prevent impurities coming into the coil. The filter can be warmed up and washed individually with pure helium gas.

Four displacement meters were installed inside a side wall of the cryostat and radial displacements of the outside surface of the coil sleeves were measured. The displacement meters are mounted at measured circumferential angles of 46 ,

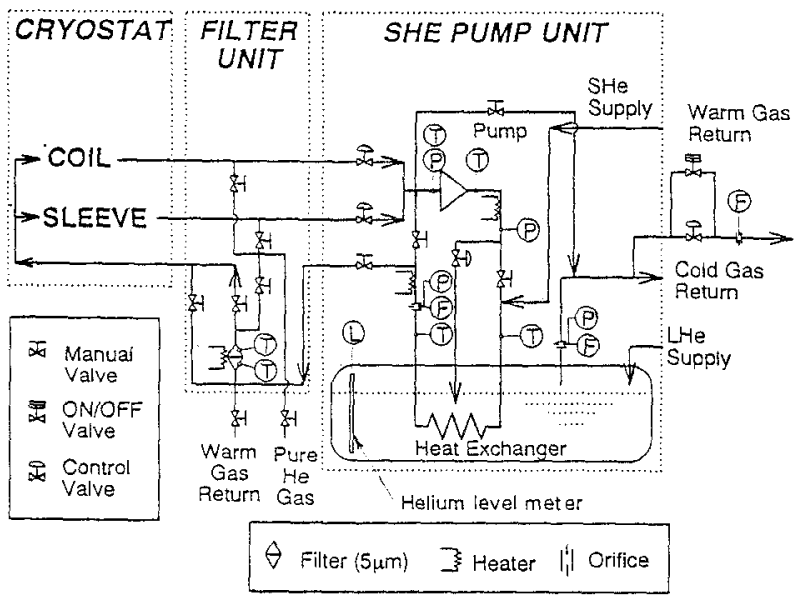

Fig 2. Cooling flow diagram and instrumentation in the supercritical helium circulating system.

134, 226 and 314 degrees, where the angle of the current feeders is zero. Acoustic emission (AE) sensors were attached onto the ground insulation of the coil.

\section{TEST RESULTS}

\section{A. Cooldown}

The cooldown was started on November 13, 1995 and continued 24 hours during 5 days a week. On every weekend, we suspended the cooldown and warmed up the cold box while keeping the coil temperature constant. Figure 3 shows the linked cooldown curves after omitting the suspended time. The cooling rates were $0.8 \mathrm{~K} / \mathrm{h}$ in the region of $290-200 \mathrm{~K}$ and $1.1 \mathrm{~K} / \mathrm{h}$ in the region of $200-100 \mathrm{~K}$. The temperature difference between inlet and outlet was successfully controlled to be less than $50 \mathrm{~K}$. The turboexpanders were started at 200 hours. The total cooldown time was about 250 hours. After the cooldown, we operated the cryogenic centrifugal pump. The pump unit generated a mass flow rate of $50 \mathrm{~g} / \mathrm{s}$, which was distributed into the coil, $40 \mathrm{~g} / \mathrm{s}$, and the sleeve, $10 \mathrm{~g} / \mathrm{s}$. The inlet temperature and pressure were $4.5 \mathrm{~K}$ and $0.9 \mathrm{MPa}$, respectively.

\section{B. Pressure Drop}

The pressure drop under the steady state flow condition was approximately $80 \mathrm{kPa}$ at $40 \mathrm{~g} / \mathrm{s}$. The pressure drop $(\Delta \mathrm{p})$ during cooldown and steady cooling is shown in Fig. 4 as the friction factor $(F)$ versus the Reynolds number. The friction factor is defined by Darcy's equation:

$$
\Delta p=F\left(\rho u^{2} / 2\right)\left(L / D_{h}\right)
$$

where $\rho, \mathrm{u}, \mathrm{L}$ and $\mathrm{D}_{\mathrm{h}}$ are the density, velocity of the coolant, length of the path, and hydraulic diameter, respectively. 
The hydraulic diameter of the conductor was calculated to be $0.5 \mathrm{~mm}$. The open and closed circles indicate the data for the first and the second cooldowns, respectively. The friction factor is larger than the Hagen-Poiseuille and Blasius equations for a smooth pipe when the Reynolds number exceeds 50 . On the other hand, Katheder's formula [5], which is a general correlation of many different conductors, agrees well with the data of IV coil.

\section{Excitation Tests}

We excited the coil several times as shown in Table III. During excitations \#2401, \#2402 and \#2701, the coil was discharged by the protection system because magnetic field leakage affected a valve control system. In \#3301, the protection system was operated again when the current just reached $20.8 \mathrm{kA}$ because the magnetic field affected a water cooling system of the power supply. It was found that the effect of magnetic field leakage on the peripheral equipment was important. After shielding the sensitive equipment from the magnetic field, the target operation was first achieved in \#3302. Figure 5 shows the current and the average voltage of seven conductor joints during this excitation. The static voltage was about $3 \mu \mathrm{V}$ at $20.8 \mathrm{kA}$. This voltage corresponds to heat generation of $0.06 \mathrm{~W}$. After \#3401, the main purpose was to measure the AC losses [6]. Though the limit of the balance voltage was set at $\pm 0.1 \mathrm{~V}$ in the quench protection system, the coil did not quench even during fast discharge. This indicates that the conductor has high stability.

\section{Radial Displacement and Acoustic Emission}

The results of displacement measurements are shown in Fig. 6. The figure indicates the total outward radial force ver sus displacement curves. The total force is the integration of every force inside the coil divided by the average coil circumference ( $\pi \times$ average diameter). The displacement is the average of four sensors, which cancels the rigid dis-

TABLE III

EXCITATION HISTORY

\begin{tabular}{|c|c|c|c|}
\hline Excitation No. & $\begin{array}{l}\text { Maximum } \\
\text { current }\end{array}$ & $\begin{array}{l}\text { Sweep rate } \\
\text { at excitation }\end{array}$ & $\begin{array}{l}\text { Sweep rate } \\
\text { at discharge }\end{array}$ \\
\hline 2301 & $5 \mathrm{kA}$ & $20 \mathrm{~A} / \mathrm{s}$ & Exponential $(\tau=17.9 \mathrm{~s})$ \\
\hline 2302 & $5 \mathrm{kA}$ & $20 \mathrm{~A} / \mathrm{s}$ & Exponential $(\tau=17.9 \mathrm{~s})$ \\
\hline 2401 & $8.1 \mathrm{kA}$ & $20 \mathrm{~A} / \mathrm{s}$ & Exponential $(\tau=17.9 \mathrm{~s})$ \\
\hline 2402 & $8.1 \mathrm{kA}$ & $20 \mathrm{~A} / \mathrm{s}$ & Exponential $(\tau=17.9 \mathrm{~s})$ \\
\hline 2701 & $9.5 \mathrm{kA}$ & $20 \mathrm{~A} / \mathrm{s}$ & Exponential $(\tau=17.9 \mathrm{~s})$ \\
\hline 3301 & $20.8 \mathrm{kA}$ & $20 \mathrm{~A} / \mathrm{s}$ & Exponential $(\tau=15.7 \mathrm{~s})$ \\
\hline 3302 & $20.8 \mathrm{kA}$ & $20 \mathrm{~A} / \mathrm{s}$ & $-60 \mathrm{~A} / \mathrm{s}$ \\
\hline 3401 & $10 \mathrm{kA}$ & $60 \mathrm{~A} / \mathrm{s}$ & $-60 \mathrm{~A} / \mathrm{s}$ \\
\hline 3501 & $15 \mathrm{kA}$ & $60 \mathrm{~A} / \mathrm{s}$ & $-60 \mathrm{~A} / \mathrm{s}$ \\
\hline 3601 & $20.8 \mathrm{kA}$ & $60 \mathrm{~A} / \mathrm{s}$ & $-60 \mathrm{~A} / \mathrm{s}$ \\
\hline 3701 & $10 \mathrm{kA}$ & $60 \mathrm{~A} / \mathrm{s}$ & Exponential $(\tau=17.9 \mathrm{~s})$ \\
\hline 3801 & $20.8 \mathrm{kA}$ & $60 \mathrm{~A} / \mathrm{s}$ & Exponential $(\tau=15.7 \mathrm{~s})$ \\
\hline 3901 & $20.8 \mathrm{kA}$ & $60 \mathrm{~A} / \mathrm{s}$ & $-60 \mathrm{~A} / \mathrm{s}$ \\
\hline
\end{tabular}

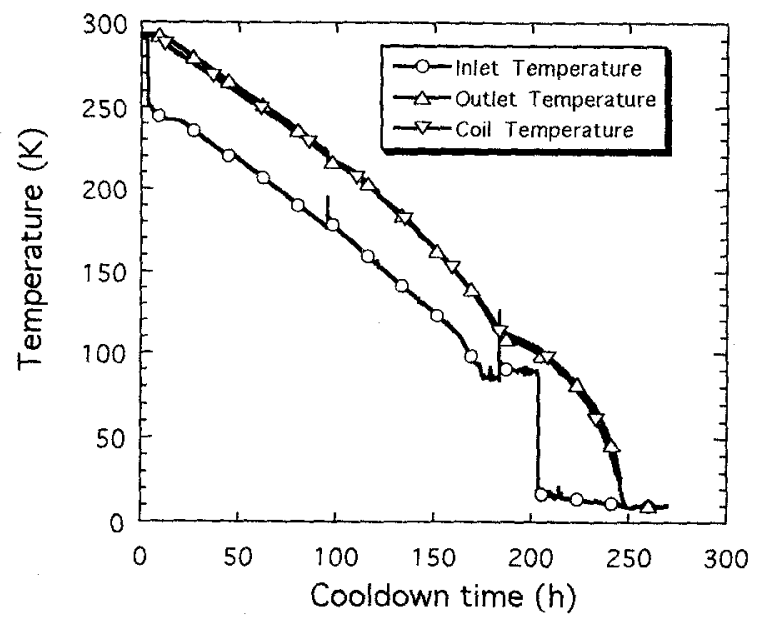

Fig. 3. Linked cooldown curves after omitting the suspended time.

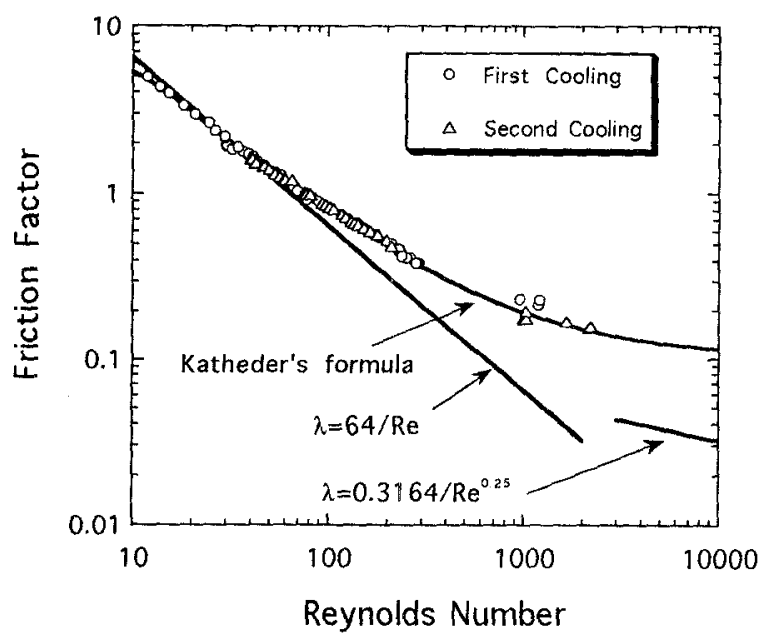

Fig. 4. Friction factor versus Reynolds number.

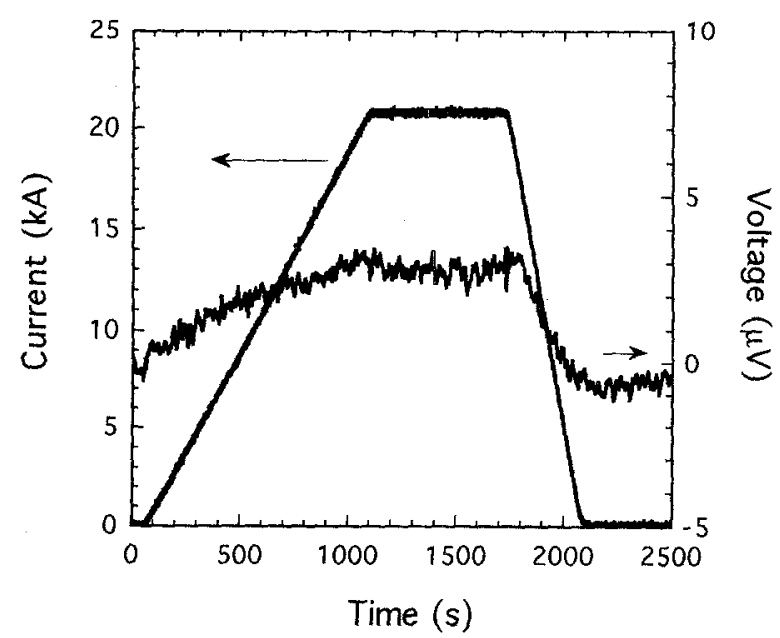

Fig. 5. Time evolution of the current and the average voltage of seven conductor joints in \#3302. 


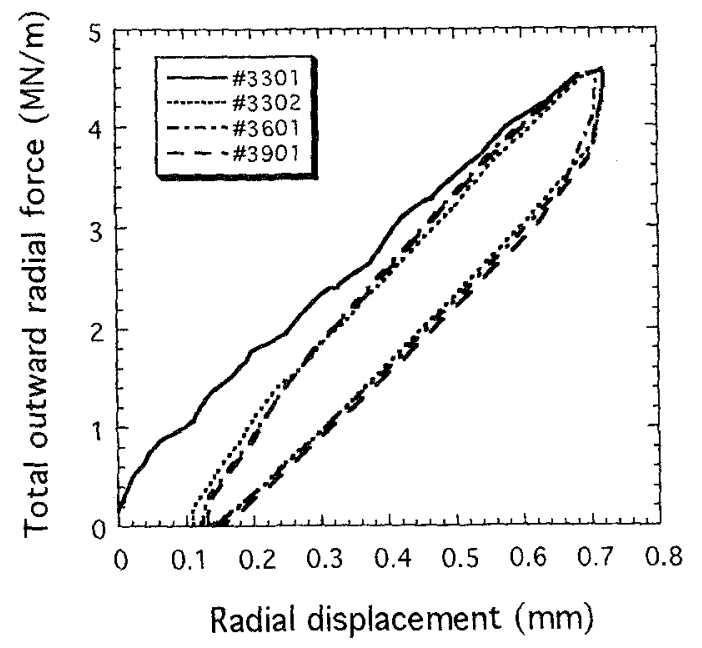

Fig. 6. Outward radial force versus displacement curves.

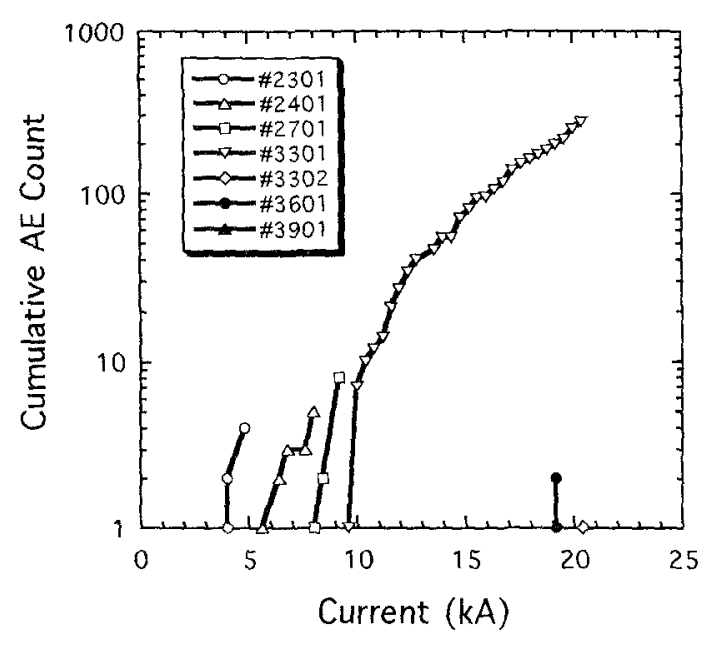

Fig. 7. Cumulative AE counts.

placement due to magnetic attraction to a building wall. From the results, the maximum displacement was found to be $0.7 \mathrm{~mm}$ at $20.8 \mathrm{kA}$. Allowable motion at the maximum current was specified to be less than $2 \mathrm{~mm}$ because the motion affects field error of the coil. The measured displacements were confirmed to be less than the allowable motion. After \#3301, a permanent displacement of $0.1 \mathrm{~mm}$ remained. This may be caused by compaction of an insulator Hysteresis of the curves may be due to sliding friction between the coil and supporting posts.

A model of a thick cylinder with inner pressure (p) was applied to estimate the coil rigidity. The outward displacement of the coil outside (d) can be calculated by the following equation:

$$
d=\left[2 r_{i}^{2} r_{o} /\left(r_{o}^{2}-r_{i}^{2}\right)\right](p / E)
$$

where $r_{i}$ and $r_{o}$ are the inner and outer radii, respectively. The total force was converted into the inner pressure by dividing by the coil height. The equivalent rigidity in the winding direction (E) was estimated about $100 \mathrm{GPa}$ by a mixture law including strands. The rigidity of strands contributes one third to the equivalent rigidity. The displacement at $20.8 \mathrm{kA}$ was then calculated as $0.77 \mathrm{~mm}$, which shows good agreement with the experimental value.

Figure 7 shows the cumulative AE counts during the excitations. It should be noted that $\mathrm{AE}$ was not observed below the maximum experienced current from the previous cycle. This is clearly an irreversible effect, which is known as the Kaiser effect [7]. The reason of the AE behavior is, therefore, considered to be the compaction of epoxy as described before. After \#3302, only a few signals were observed. This confirms that the force did not damage the coil.

\section{CONCLUSIONS}

The excitation tests of a single inner vertical coil for the Large Helical Device were carried out to confirm its performance. The coil was successfully energized up to the specified current without quench. The heat generation of a joint was estimated to be $0.06 \mathrm{~W}$ at $20.8 \mathrm{kA}$. The maximum outward radial displacement was $0.7 \mathrm{~mm}$, which is within the design specification. The equivalent rigidity of the coil could be estimated from the mixture law including strands. The AE signals proved that the electro-magnetic force did not damage the coil although the coil was free to move.

\section{ACKNOWLEDGMENT}

The authors would like to thank many staff members in NIFS who have been involved in the long-term operation of the refrigerator and the test facility. We are also grateful to Fuji Electric Co., Ltd. and IHI Co., Ltd. for their help to operate the superconducting bus line and the cryogenic pump.

\section{REFERENCES}

[1] O. Motojima, et al., "Physics and engineering design studies on the Large Helical Device," Fusion Engineering Design, Vol. 20, pp. 3-14, 1993.

[2] K. Takahata, et al., "Cooldown performance of an inner venical field coil for the Large Helical Device," IEEE Trans. on Magn., Vol. 32, pp. 2252-2255, 1996.

[3] S. Hanawa, et al., "Development of a superconducting joint technique between CIC conductors for poloidal coil of Large Helical Device (LHD)," IEEE Trans. on Appl. Supercond., Vol, 5, pp. 757-760, 1995.

[4] K. Nakamoto, et al., "Design and fabrication of forced-flow superconducting poloidal coils for the Large Helical Device," FUSION TECHNOLOGY, Vol. 2, pp. 909-912, 1994.

[5] H. Katheder, "Optimum themohydraulic operation regime for cable in conduit supenconductors (CICS)," Cryogenics, Vol. 34, pp. 595-598, 1994.

[6] T. Mito, et al., "AC loss measurements of the experiments on a single inner vertical coil (EXSIV) for the Large Hel ical Device," LDB-12, this conference.

[7] N. Maeda and Y. Iwasa, "Heat generation from epoxy cracks and bond failures," Cryogenics, Vol. 22, pp. 473-476, 1982. 